\title{
Pediatric adherence to antiretroviral therapy in resource-poor settings: challenges and future perspectives
}

\author{
"By the end of 2007, more than 2 million children under 15 years of age worldwide \\ were living with HIV, $90 \%$ in sub-Saharan Africa."
}

The HIV pediatric epidemic in low-income countries is still growing with an increasing impact on children. By the end of 2007, more than 2 million children under 15 years of age worldwide were living with HIV, $90 \%$ in subSaharan Africa. In that year alone, 370,000 children were newly infected and 270,000 died. AIDS has become one of the leading causes of mortality among children under the age of 5 years in developing countries [101]. In the absence of combination antiretroviral therapy (cART), $52 \%$ of children infected with perinatally acquired HIV infection will die by the age of 2 years [1].

Numerous studies have confirmed the clinical efficacy and feasibility of cART in HIVinfected adults in Africa $[2,3]$ but, to date, resources and programs targeting HIV-infected children in resource-poor settings remain limited. Even though the use of cART to treat children has increased in recent years in subSaharan Africa, less than $15 \%$ of children needing cART in Africa currently receive it [102]. It is estimated that more than 780,000 children are in need of cART in low- and middle-income countries [103]. When made accessible, treatment for children in this context has proved highly effective [4]. Studies have found the survival probability at 12 months for children on cART to be more than $95 \%$ in settings in sub-Saharan Africa [5] and Asia [6]. Education and adherence counseling are therefore essential components of cART and adherence in HIV-infected children is critical to the success of cART.

\section{Specificities \& challenges of pediatric cART adherence}

The issue of adherence in children requires particular attention because of its specificities in comparison to adults as:

- Children may not be aware of their HIV status;
- Younger children depend on caregivers for receiving drugs;

- According to their age, adherence issues differ in terms of the cognitive development, level of maturity and feelings of the children;

- There are currently very few adequate pediatric formulations, raising palatability difficulties;

- There is often difficulties in the family structure with fewer adults/parents to care for the infected child (orphans and vulnerable children);

- The frequent lack of healthcare professionals with adequate childcare competencies.

Currently, several arguments are in favor of the early and systematic initiation of antiretroviral treatment among HIV-infected children at the earliest convenience to improve their survival, as reported in the Children with HIV Early Antiretroviral Therapy (CHER) Trial [7]. This led to a revision of the WHO pediatric guidelines in 2008 recommending that HIV-infected infants are treated systemically, irrespective of their clinical stage [104]. In low-income countries, it is crucial to assess public health strategies of early HIV diagnosis and lifelong cART targeted to HIV-infected infants. This early initiation of cART in infants also raises questions concerning long-term effects in terms of safety, efficacy and resistance, but also adherence.

Successful treatment of HIV requires drug adherence rates of $95 \%[8]$. Even moderate nonadherence to cART is associated with compromised health status and significant disease progression [9]. On the contrary, good adherence minimizes the likelihood of developing resistance to a particular drug or class of drugs [10], a factor of great importance in resource-limited settings with few treatment options.

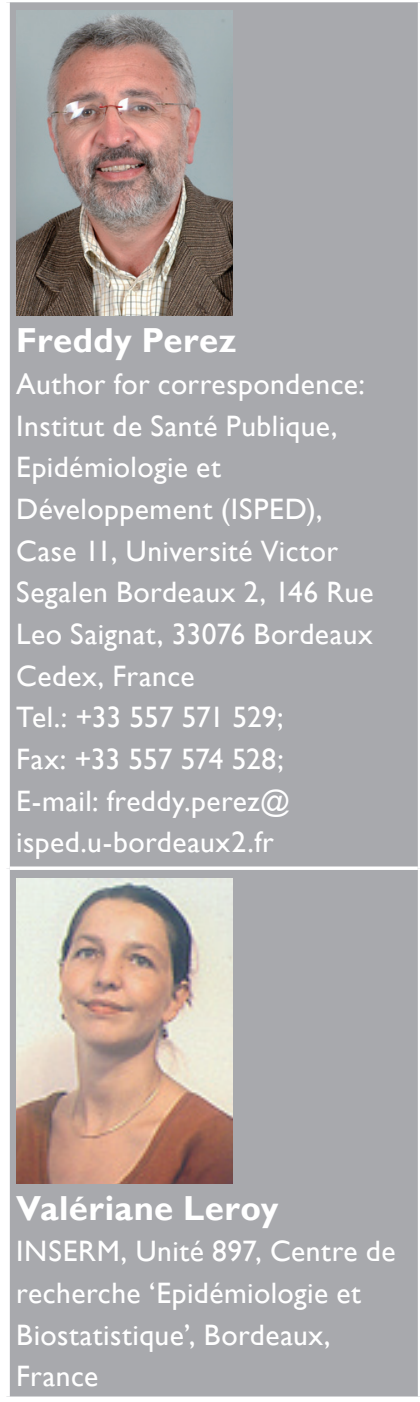

future ${ }_{\text {medine }}$ fss 


\section{"Successful treatment of HIV requires drug adherence rates of $95 \%$. Even moderate nonadherence to CART is associated with compromised health status and significant disease progression."}

Although adherence in adults has been well documented in developed and developing countries [11,12], less attention has been given to cART adherence among children. The need for near-perfect adherence to lifelong therapy from an early age has been identified as a major challenge in the administration of cART to HIV-infected children in a context where there is a crucial lack of accessibility to antiretroviral drugs [13].

Sustaining high rates of adherence in pediatric care is challenging for children receiving the treatment, their caregivers, as well as healthcare providers. Drug regimens include multiple medicines that may have complex dosing schedules often requiring that patients take numerous pills multiple times a day with specific timing and food restrictions or requirements and adverse effects resulting in poor tolerability [13]. Furthermore, discrimination and stigma may prevent caregivers from disclosing the child's HIV status with family members or others [14].

\section{Assessing adherence to pediatric cART}

Regardless of the benefits of cART in treating pediatric HIV infection, estimates of adherence to cART have been reported to be suboptimal amongst children within high-income countries [15]. This is largely dependent on the definition of adherence, the method used to assess it and the study setting [16].

\section{- Methods of measurement}

There have been several different methods to measure pediatric cART adherence in resource-limited settings, including self or caregiver reports [17-19], pill counts [20], pharmacy records [21], clinic attendance [22], therapeutic drug monitoring [23] and directly observed therapy [24]. Very few studies have compared clinical indicators (viral loads or CD4 counts) with measured adherence [5], or used electronic drug monitoring as a measure of adherence in this context [25]. It is difficult to estimate cART adherence in children with precision since the measurement methods are not standardized. Data from studies of pediatric cART adherence fail to provide definitive guidelines or to identify any gold standard in terms of assessment methods.

The limitations of any single assessment strategy suggest possible benefits of using multiple methods. It is often recommended to associate the patient's and/or caregiver's declaration of adherence with virological outcomes [26]. Collecting information on adherence from multiple sources of information has also been suggested; nevertheless, few studies have validated adherence assessment strategies [18].

It is notable that because varying definitions of adherence among studies and different quantifying adherence strategies have been used, it has not been possible to produce a pooled estimate on pediatric adherence in developing countries. How adherence is assessed, how optimal adherence is defined (cut off points of 90, 95 or 100) and how findings are reported have varied considerably between studies.

In resource-limited settings, pediatric cART adherence assessment is often restricted to the patient caregiver (as a proxy) or child/youth self-report and pill counts because of financial constraints [17]. Self-reported/caregiver adherence tends to overestimate the prevalence of adherence due to social desirability or recall bias factors [27]. In this sense, Farley et al. found self-report to be unreliable when compared with electronic measurement [28].

More objective measures of adherence such as unannounced pill count and medication event monitoring system (MEMS) tend to be more sensitive to lapses in adherence $[9,18]$. However, such measures are not feasible in resource-limited settings with large-scale programs. Davies et al. showed that although clinic-based medication measures may not be as sensitive as unannounced home-based measures or MEMS monitoring to detect poor adherence, they are still strongly predictive of virological response [29].

\section{Level of adherence}

Despite its importance in ensuring treatment success, a limited number of studies have been carried out among HIV-infected children concerning adherence to cART in resource-poor settings to date [17-19]. In a systemic review from low-income countries, Vreeman et al. presented estimates of cART adherence ranging from 49 to $100 \%$, with the majority of studies reporting an adherence of greater than $75 \%$ [30]. In addition, recent prospective studies have highlighted that good adherence to cART in resource-poor settings is achievable with $80-90 \%$ of children obtaining average medication return adherence greater than $95 \%$, resulting in 84 and $80 \%$ virological suppression at 6 and 12 months, respectively [6,29]. 


\section{Correlates \& determinants of pediatric adherence to CART in resource-poor settings}

In order to facilitate adherence to cART and to improve outcomes of cART in HIV-infected children it becomes necessary to identify correlates and factors that influence adherence and determine possible interventions to improve cART adherence in children.

Several studies have recently been reported that describe the benefits of adherence and have identified nonadherence factors in children in resource-poor settings [17,19]. Specific findings vary from study to study, which is, in part, due to the different samples and measures used.

Child adherence is thwarted by multiple barriers including individual and broader structural factors related to patient and family/caregiver, medication and healthcare delivery systems [31]. Knowing and understanding these factors is helpful in identifying where and how to improve adherence to cART amongst children.

\section{- Family/caregiver correlates}

Family/caregiver correlates are crucial to pediatric adherence because infants and younger children depend almost entirely on a caregiver to administer medication [9]. Various studies in resource-limited settings have confirmed the crucial role the caregiver has in pediatric cART adherence. Characteristics of the child's familial and socioeconomic environment has been shown to be significantly associated with nonadherence, including low parental education and disorganized families [32]. Furthermore, caregiver psychosocial factors, such as presence of anxiety, depression, fear of disclosure of HIV-positivity to family members, the perception and belief in the efficacy of the treatment, dosing self-efficacy, knowledge of the disease and cART, along with the financial burden and associated costs of medical treatment, have been identified as significant predictors of child adherence $[17,27]$

Regarding patient correlates, age (especially infancy and adolescence), refusal of treatment, knowledge of HIV status, clinical stage and changes in health status have also been identified as relevant issues in relation to adherence to cART in pediatric patients [33]. A study in Uganda identified complete disclosure and strong parental relationships as factors related to good adherence [19]. In addition, structural factors such as poverty and stigma were identified barriers to adherence. Complete parental disclosure helps to motivate HIV-infected children to understand HIV infection and to make sense of disease-related experiences and the importance of adherence [34].

For adolescents, issues such as body modifications induced by puberty, authority problems, depression, aggressiveness and accession to sexuality are only some of the difficulties that will increase the problem of adherence. This a critical period with potential adherence fatigue leading to treatment failure [35].

An estimated 15 million children are orphaned as a result of HIV, with $80 \%$ of these orphans living in sub-Saharan Africa [101]. Contextual factors such as orphan status (child who lost one or both parents) may influence pediatric adherence to cART. Prior research concerning orphan status and its association with cART nonadherence has been inconsistent [33,36,37]. In a recent study in Uganda, orphans were more likely to initiate cART at an older age than non-orphans [38]. There is a need to better understand the relationship between pediatric cART adherence and the complex context of the death of one or both parents, and target those at risk for nonadherence so as to ensure timely cART access.

\section{- Medication-related factors}

Proposed medication- or treatment-related factors that likely complicate pediatric adherence include complex regimens, high pill burden, complex dietary considerations, storage requirements and long-term toxicity. Other factors to consider are the characteristics of the available formulations of drugs such as taste, size of pills, availability of liquid formula, storage requirements and adverse effects [31]. To date there are a limited number of antiretrovirals approved worldwide for pediatric use, and these related factors become crucial in determining child's adherence to cART.

\section{- Healthcare delivery system related factors}

The healthcare system has an important role in determining adherence. Decentralization of pediatric HIV care treatment from third-line health services to district level and the provision of a continuum of services within a family approach are major challenges to improve coverage of pediatric cART in resource-poor settings. This requires monitoring medication adherence, providing strategies to enhance adherence as well as establishing a reliable drug distribution system. Trust and communication with health providers as well as using directly observed therapy

\section{"It is notable that because varying definitions of adherence among studies and different quantifying adherence strategies have been used, it has not been possible to produce a pooled estimate on pediatric adherence in developing countries."}


or watching children taking therapy have been reported to improve adherence [24]. These interventions require trained and committed healthcare staff and time to be spent with patients and their caregivers. Further data are needed to evaluate the long-term sustainability of this approach.

\section{Interventions to promote pediatric adherence to CART in resource-poor settings}

Even though there have been studies of factors related to adherence, very little work has been carried out to identify ways to improve cART adherence in children generally and in resourcepoor settings in particular. In addition, limited information has been reported on the evaluation of adherence-promoting interventions. At present, a few feasible strategies to encourage and improve pediatric cART adherence that can be applied in resource-poor settings have been identified.

\section{- Interventions aimed to reduce the} barriers to adherence

"The high rates of HIV-positive caregivers indicates that it is imperative to transform the pediatric treatment model from child orientated to family orientated in order to protect the integrity of care giving structures."
Simplifying treatment regimens when possible and tailoring CART regimens to the daily activities of the child and his/her family may enhance patient adherence to therapy [31]. Developing fixed-dose combinations and thus lowering pill burden that are appropriately formulated for children is a priority [39]. Palatable pediatric syrups and appropriate low-dose tablet formulations remain all too rare. There is an urgent need for the development of simplified pediatric antiretroviral regimens. With this aim, once-daily regimens are promising options that need to be developed [40].

Disclosure of HIV status to children over 6 years of age and implicating children in the decision-making process regarding therapy modifications has been reported to enhance the child's cooperation with treatment [17]. There is a need to assess the nature of the parental relationship and consider support activities aimed at caregivers on how to announce the serological status to the child [41].

\section{- Interventions aimed to support adherence}

Before treatment begins, educating, counseling and giving continuous psychological and social support to parents and/or caregivers as well as patients is required through the implementation of programs to support adherence for HIVpositive children in need of cART [42]. Assessing regimen knowledge at periodic intervals to identify families at risk for nonadherence so that educational interventions can be tailored toward this group and using directly observed therapy through involvement of community workers are some of the strategies that can result in improved pediatric adherence to cART [24].

Owing to expanding services for cART in resource-poor settings, there is a need to address severe human resource shortage. Task-shifting strategies (delegation of health service responsibilities from higher to lower cadres of healthcare staff) through the training of community health workers and lay counselors as adherence support workers should be considered. Recent implementation of this strategy has proved feasible in HIV-testing centers [43]. However, there will be a need to assure the provision of quality assurance through ongoing supervision. There is very limited evidencebased data on how task shifting influences the quality, safety, acceptability and impact of cART interventions in sub-Saharan Africa. Operational research in this field is still needed [44].

The establishment of a long-term relationship between children, families and clinic staff has been reported as a key intervention [45]. In this sense, adherence programs that include strategies to enhance and facilitate communication between the family and care providers can help overcome language and cultural barriers.

The high rates of HIV-positive caregivers indicates that it is imperative to transform the pediatric treatment model from child orientated to family orientated in order to protect the integrity of care giving structures. This familyorientated approach needs to be free of charge or associated with strategies to identify indigents, and able to support minimal financial needs [46].

During treatment, adherence should be repeatedly monitored and promoted whenever needed. Several psychosocial interventions such as disclosure or support groups could play a role; on the other hand, there are also different tools that could assist patients with taking treatment (e.g., pill boxes and calendars, among others).

\section{Priorities for future research on pediatric CART in resource-poor settings}

Promoting adherence is a multidisciplinary challenge that raises research and programmatic perspectives.

\section{Research issues}

Below are a number of directions that should be considered in future research on pediatric adherence to cART in resource-poor settings. 
- Systemic meta-studies that focus on African pediatric cART adherence should be encouraged. This might provide a more complete understanding of the problem of pediatric adherence;

- As has been recommended by others [31], estimates of adherence need to be based on two or more assessment techniques. To date, the use of multiple measures of adherence is rare $[18,29]$. Therefore, comparison between self-report, objective measures and clinical indicators are needed to identify the most cost-effective assessment strategies [47]. In addition to this, standardization of assessment methods and definitions of adherence will facilitate the comparison of research results originating from resource-poor settings. A low-cost, reliable, replicable and easy-to-use tool is needed to measure adherence specifically in children in routine clinical follow-up;

- Most studies have reported adherence correlates by cross-sectional designs. Future research should incorporate prospective studies to investigate the variability and consistency of adherence repeatedly over time, as well as predictors of short- and long-term adherence [25,29];

- Currently, cART is mainly initiated during the advanced stages of HIV in African children. Recent data have shown the benefits of early cART in children [7]. There is an urgent need to evaluate early pediatric treatment adherence;
- Simplification of long-term antiretroviral treatment relevant in children could focus on a once-daily strategy easier to maintain for a lifelong treatment. This type of approach needs to be further investigated in infants and children.

\section{- Programmatic issues}

A reliable tool of adherence that can be adapted to children less than 3 years of age, who are highly dependent on caregivers and need specific drug formulations is urgently needed.

\section{Conclusion}

With more than 2 million children under the age of 15 currently living with HIV, interventions to enhance pediatric cART adherence at the patient, family and healthcare level in lowincome countries are an urgent priority. A major challenge for developing countries is adapting the healthcare infrastructure and models for the management of chronic diseases. Operational research that enhances our understanding of cART adherence will be critical.

\section{Financial \& competing interests disclosure}

The authors have no relevant affiliations or financial involvement with any organization or entity with a financial interest in or financial conflict with the subject matter or materials discussed in the manuscript. This includes employment, consultancies, honoraria, stock ownership or options, expert testimony, grants or patents received or pending, or royalties.

No writing assistance was utilized in the production of this manuscript.

\author{
"A major challenge for \\ developing countries is \\ adapting the healthcare \\ infrastructure and models for \\ the management of chronic \\ diseases. Operational \\ research that enhances our \\ understanding of ART \\ adherence will be critical."
}

\section{Bibliography}

Papers of special note have been highlighted as: - of interest

-1" of considerable interest

1 Newell ML, Coovadia H, Cortina-Borja M, Rollins N, Gaillard P, Dabis F: Mortality of infected and uninfected infants born to HIV-infected mothers in Africa: a pooled analysis. Lancet 364(9441), 1236-1243 (2004).

2 Toure S, Kouadio B, Seyler C et al.: Aconda study group. Rapid scaling-up of antiretroviral therapy in 10,000 adults in Côte d'Ivoire: 2-year outcomes and determinants. AIDS 22(7), 873-882 (2008).

3 Bussmann H, Wester CW, Ndwapi N et al:: Five-year outcomes of initial patients treated in Botswana's National Antiretroviral Treatment Program AIDS 22(17), 2303-2311 (2008).
4 George E, Noël F, Bois G et al.: Antiretroviral therapy for HIV-1-infected children in Haiti. J. Infect. Dis. 195(10), 1411-1418 (2007).

5 Reddi A, Leeper SC, Grobler AC et al.: Preliminary outcomes of a paediatric highly active antiretroviral therapy cohort from KwaZulu-Natal, South Africa. BMC Pediatr. 7, 13 (2007).

6 Puthanakit T, Oberdorfer A, Akarathum N et al.: Efficacy of highly active antiretroviral therapy in HIV-infected children participating in Thailand's National Access to Antiretroviral Program. Clin. Infect. Dis. 41(1), 100-107 (2005).

7 Violari A, Cotton MF, Gibb DM et al.: Early antiretroviral therapy and mortality among HIV-infected infants. N. Engl. J. Med. 359(21), 2233-2244 (2008).
-1" First randomized, controlled study to address the issue of when to start combination antiretroviral therapy (cART) in children.

8 Paterson DL, Swindells S, Mohr J et al.: Adherence to protease inhibitor therapy and outcomes in patients with HIV infection. Ann. Intern. Med. 133(1), 21-30 (2000).

9 Steele RG, Grauer D: Adherence to antiretroviral therapy for pediatric HIV infection: review of the literature and recommendations for research. Clin. Child Fam. Psychol. Rev. 6(1), 17-30 (2003).

10 Sethi AK, Celentano DD, Gange SJ, Moore RD, Gallant JE: Association between adherence to antiretroviral therapy and human immunodeficiency virus drug resistance. Clin. Infect. Dis. 37(8), 1112-1118 (2003). 
11 Oyugi JH, Byakika-Tusiime J, Charlebois ED et al:: Multiple validated measures of adherence indicate high levels of adherence to generic HIV antiretroviral therapy in a resource-limited setting. J. Acquir. Immune Defic. Syndr. 36(5), 1100-1102 (2004).

12 McNabb J, Ross JW, Abriola K, Turley C, Nightingale CH, Nicolau DP: Adherence to highly active antiretroviral therapy predicts virologic outcome at an inner-city human immunodeficiency virus clinic. Clin. Infect. Dis. 33(5), 700-705 (2001).

13 Eley B, Nuttall J: Antiretroviral therapy for children: challenges and opportunities. Ann. Trop. Paediatr. 27(1), 1-10 (2007).

14 Abadía-Barrero CE, Castro A: Experiences of stigma and access to HAART in children and adolescents living with HIV/AIDS in Brazil. Soc. Sci. Med. 62(5), 1219-1228 (2006).

15 Martin S, Elliott-DeSorbo DK, Wolters PL et al.: Patient, caregiver and regimen characteristics associated with adherence to highly active antiretroviral therapy among HIV-infected children and adolescents. Pediatr. Infect. Dis. J. 26(1), 61-67 (2007).

16 Simoni JM, Montgomery A, Martin E, New M, Demas PA, Rana S: Adherence to antiretroviral therapy for pediatric HIV infection: a qualitative systematic review with recommendations for research and clinical management. Pediatrics 119(6), E1371-E1383 (2007).

17 Polisset J, Ametonou F, Arrive E, Aho A, Perez F: Correlates of adherence to antiretroviral therapy in HIV-infected children in Lomé, Togo, West Africa. AIDS Behav. 13(1), 23-32 (2009).

18 Nabukeera-Barungi N, Kalyesubula I, Kekitiinwa A, Byakika-Tusiime J, Musoke P: Adherence to antiretroviral therapy in children attending Mulago Hospital, Kampala. Ann. Trop. Paediatr. 27(2), 123-131 (2007).

19 Bikaako-Kajura W, Luyirika E, Purcell DW et al: : Disclosure of HIV status and adherence to daily drug regimens among HIV-infected children in Uganda. AIDS Behav. 10 (Suppl. 4), S85-S93 (2006).

20 Eley B, Nuttall J, Davies MA et al.: Initial experience of a public sector antiretroviral treatment programme for HIV-infected children and their infected parents. S. Afr. Med. J. 94(8), 643-646 (2004).

21 Evans-Gilbert T, Pierre R, Steel-Duncan JC et al.: Antiretroviral drug therapy in HIV-infected Jamaican children. West Indian Med. J. 53(5), 322-326 (2004).

22 Natu SA, Daga SR: Antiretroviral therapy in children: Indian experience. Indian Pediatr. 44(5), 339-343 (2007).
23 Bunupuradah T, Wannachai S, Chuamchaitrakool A et al.: Use of tastemasking product, FLAVORx, to assist Thai children to ingest generic antiretrovirals. AIDS Res. Ther. 3, 30 (2006).

24 Myung P, Pugatch D, Brady MF et al.: Directly observed highly active antiretroviral therapy for HIV-infected children in Cambodia. Am. J. Public Health 97(6), 974-977 (2007).

25 Müller AD, Bode S, Myer L, Roux P, von Steinbüchel N: Electronic measurement of adherence to pediatric antiretroviral therapy in South Africa. Pediatr. Infect. Dis. J. 27(3), 257-262 (2008).

- Study showing the feasibility of using a medication event monitoring system as a measure of adherence to cART in resource-poor settings.

26 Williams PL, Storm D, Montepiedra G et al:: Predictors of adherence to antiretroviral medications in children and adolescents with HIV infection. Pediatrics 118(6), e1745e1757 (2006).

27 Mellins CA, Brackis-Cott E, Dolezal C, Abrams EJ: The role of psychosocial and family factors in adherence to antiretroviral treatment in human immunodeficiency virus-infected children. Pediatr. Infect. Dis. J. 23(11), 1035-1041 (2004).

28 Farley J, Hines S, Musk A, Ferrus S, Tepper V: Assessment of adherence to antiviral therapy in HIV-infected children using the medication event monitoring system, pharmacy refill, provider assessment, caregiver self-report, and appointment keeping. J. Acquir. Immune Defic. Syndr. 33(2), 211-218 (2003).

29 Davies MA, Boulle A, Fakir T, Nuttall J, Eley B: Adherence to antiretroviral therapy in young children in Cape Town, South Africa, measured by medication return and caregiver self-report: a prospective cohort study. $B M C$ Pediatr. 8, 34 (2008).

30 Vreeman RC, Wiehe SE, Pearce EC, Nyandiko WM: A systematic review of pediatric adherence to antiretroviral therapy in low- and middle-income countries. Pediatr. Infect. Dis. J. 27(8), 686-691 (2008).

"I- First complete review of pediatric adherence to cART in resource-limited settings.

31 Pontali E: Facilitating adherence to highly active antiretroviral therapy in children with HIV infection: what are the issues and what can be done? Paediatr. Drugs 7(3), 137-149 (2005).

32 Wachholz NI, Ferreira J: Adherence to antiretroviral therapy in children: a study of prevalence and associated factors. Cad. Saude Publica 23(Suppl. 3), S424-S434 (2007).
33 Giacomet V, Albano F, Starace F et al.: Adherence to antiretroviral therapy and its determinants in children with human immunodeficiency virus infection: a multicentre, national study. Acta Paediatr. 92(12), 1398-1402 (2003).

34 Blasini I, Chantry C, Cruz C et al.: Disclosure model for pediatric patients living with HIV in Puerto Rico: design, implementation, and evaluation. J. Dev. Behav. Pediatr. 25(3), 181-189 (2004).

35 Parsons GN, Siberry GK, Parsons JK et al.: Multidisciplinary, inpatient directly observed therapy for HIV-1-infected children and adolescents failing HAART: a retrospective study. AIDS Patient Care STDS 20, 275-284 (2006).

36 Nyandiko WM, Ayaya S, Nabakwe E et al.: Outcomes of HIV-infected orphaned and nonorphaned children on antiretroviral therapy in western Kenya. J. Acquir. Immune Defic. Syndr. 43(4), 418-425 (2006).

37 Vreeman RC, Wiehe SE, Ayaya SO, Musick BS, Nyandiko WM: Association of antiretroviral and clinic adherence with orphan status among HIV-infected children in Western Kenya. J. Acquir. Immune Defic. Syndr. 49(2), 163-170 (2008).

38 Kiboneka A, Wangisi J, Nabiryo C et al.: Clinical and immunological outcomes of a national paediatric cohort receiving combination antiretroviral therapy in Uganda. AIDS 22(18), 2493-2499 (2008).

- Study evaluating clinical and immunological outcomes of pediatric patients receiving cART and showing the need to ensure early access to cART for orphans and vulnerable populations.

39 L'homme RF, Kabamba D, Ewings FM et al. Nevirapine, stavudine and lamivudine pharmacokinetics in African children on paediatric fixed-dose combination tablets. AIDS 22(5), 557-565 (2008).

40 Willig JH, Abroms S, Westfall AO et al.: Increased regimen durability in the era of once-daily fixed-dose combination antiretroviral therapy. AIDS 22(15), 1951-1960 (2008).

41 Wiener L, Mellins CA, Marhefka S, Battles HB: Disclosure of an HIV diagnosis to children: history, current research, and future directions. J. Dev. Behav. Pediatr. 28(2), 155-166 (2007).

42 Nicholson O, Mellins C, Dolezal C, Brackis-Cott E, Abrams EJ: HIV treatment-related knowledge and selfefficacy among caregivers of HIV-infected children. Patient Educ. Couns. 61(3), 405-410 (2006). 
43 Torpey KE, Kabaso ME, Mutale LN et al.: Adherence support workers: a way to address human resource constraints in antiretroviral treatment programs in the public health setting in Zambia. PLoS ONE 3(5), e2204 (2008).

"I- Among the first studies to show the feasibility of delegating adherence counseling tasks to lay cadres (community health volunteers who are trained to provide a specific service) without compromising the quality of counseling.

44 van Griensven J, De Naeyer L, Uwera J, Asiimwe A, Gazille C, Reid T: Success with antiretroviral treatment for children in Kigali, Rwanda: experience with health center/ nurse-based care. BMC Pediatr. 8, 39 (2008).

45 Brackis-Cott E, Mellins CA, Abrams E, Reval T, Dolezal C: Pediatric HIV medication adherence: the views of medical providers from two primary care programs. J. Pediatr. Health Care 17(5), 252-260 (2003).
46 Reddi A, Leeper SC, Sunpath H: Pediatric highly active antiretroviral therapy in Africa: potential benefits of a family-centered model. J. Infect. Dis. 198(6), 938-939 (2008).

47 Wiener L, Riekert K, Ryder C, Wood LV: Assessing medication adherence in adolescents with HIV when electronic monitoring is not feasible. AIDS Patient Care STDS 18(9), 527-538 (2004).

\section{- Websites}

101 UNAIDS. Report on the global AIDS epidemic 2008 (Accessed 10 January 2009). www.unaids.org/en/KnowledgeCentre/ HIVData/GlobalReport/2008/2008_ Global_report.asp

102 WHO. Toward universal access: scaling up priority interventions in the health sector: progress report, April 2007 (Accessed 15 December 2008). www.who.int/hiv/mediacentre/universal_ access_progress_report_en.pdf
103 WHO. Children and AIDS. Third stocktaking report 2008 (Accessed 2 February 2009).

www.uniteforchildren.org/knowmore/files/ StocktakingReport08_Full_110708.pdf

104 WHO. Report of the WHO Technical Reference Group, pediatric HIV/ART care guideline group meeting. Revised treatment recommendations for infants (Accessed 2 February 2009). www.who.int/hiv/pub/paediatric/WHO_ Paediatric_ART_guideline_rev_ mreport_2008.pdf

" - Revised WHO guidelines on pediatric HIV care and treatment covering: when to start ART in infants, what antiretroviral medicines should be used to treat infants and when the optimum time is for testing infants born to women with HIV. 